Mögliche Schäden durch den Klimawandel

\title{
Makroökonomische Bewertung von Extremwetterereignissen in Deutschland
}

\begin{abstract}
Extremwetterereignisse werden infolge des Klimawandels zukünftig häufiger auftreten. Die deutsche Volkswirtschaft ist aufgrund ihrer hochtechnischen Ausdifferenzierung potenziell besonders anfällig. Zur Planung von Anpassungsmaßnahmen müssen diese Risiken auf gesamtwirtschaftlicher Ebene abgebildet und bewertet werden. Von Ulrike Lehr und Anne Nieters
\end{abstract}

S eit den 1970er-Jahren nehmen Extremwetterereignisse wie Stürme, Überschwemmungen oder Hitzewellen zu und steigen die dadurch verursachten Schäden (Münchner Rück 2013). Dies ist, auch nach den jüngsten Szenarien des Zwischenstaatlichen Ausschusses über Klimaveränderung (IPCC 2014), eine der möglichen Ausprägungen des Klimawandels in Mitteleuropa und kann die wirtschaftliche Leistungsfähigkeit der deutschen Volkswirtschaft in Zukunft beeinflussen (Rahmstorf/Schellnhuber 2006).

\section{Methodisches Vorgehen}

Bislang wurden zur Abschätzung von Klimawandelfolgen technisch-physikalische Modelle oder Integrated Assessment Modelle eingesetzt. Erstere haben den Vorteil, dass sie die physikalischen Zusammenhänge eines Hochwassers und seiner Ausbreitung hervorragend abbilden, letztere versuchen, das gesamte Klima-Ökonomie-System abzubilden, mit den diesem umfassenden Ansatz geschuldeten Vereinfachungen. Hier wird ein dritter Weg gegangen und ein bisher für die Wirkungsanalyse von umwelt- und klimapolitischen Instrumenten genutztes makroökonometrisches Modell erweitert und ertüchtigt, sodass Schäden durch Extremwetterereignisse abbildbar sind. Das eingesetzte Modell PANTA RHEI ist in der Literatur ausführlich dokumentiert (Lehr et al. 2012; Ahlert et al. 2009). PANTA RHEI wurde in der Vergangenheit zum Beispiel zur Analyse der Beschäftigungswirkungen der Erneuerbaren Energien in Deutschland (Lehr/Ulrich 2014), der Energieeffizienz (Lutz/Lehr 2014) oder der Energieszenarien für das deutsche Energiekonzept (Prognos/EWI/GWS 2010) eingesetzt. In diesen Untersuchungen stehen die Auswirkungen von Preisänderungen oder veränderten Investitionsentscheidungen und die jeweiligen ökonomischen Anpassungsreaktionen im Vordergrund. Das Modell bildet die Volkswirtschaft in einem geschlossenen Rahmen in tiefer sektoraler Disaggregation ab und zeichnet sich durch auf Zeitreihen basierenden, ökonometrisch geschätzten Nachfragefunktionen aus. Diese Konstruktionsprinzipien wurden in den neuen Modulen zur Abbildung der Folgen von Extremwetterereignissen beibehalten. Allerdings liegen zu Extremwetterereignissen in Deutschland keine Zeitreihen vor. Kosten und Schäden vergangener Hochwasser- und Hitzeereignisse wurden daher vor allem von den Auswirkungen des Oder/ElbeHochwassers 2002 und der Hitzewelle 2003 abgeleitet, die als Referenzereignisse dienen. In einigen Fällen war dies nicht möglich und es wurde auf Daten anderer Extremwetterereignisse oder Literaturwerte zurückgegriffen.

Wer aber ist wie von Extremwettern betroffen? Hier ist die detaillierte Modellierung einzelner Wirtschaftszweige vorteilhaft. So wirkt extreme Hitze auf Unternehmen, die auf Kühlung ihrer Prozesse angewiesen sind, wie die Energiewirtschaft, bis hin zur Produktionsunterbrechung oder zum Produktionsausfall. Unternehmen, die in Flussgebieten liegen, müssen hochwasserbedingt auf Ausfälle von Infrastruktur bei der Zulieferung von Vorleistungen reagieren oder sogar Teile ihres Kapitalstocks wie Maschinenhallen und Produktionsanlagen aufgeben, beziehungsweise ersetzen. Falls die Unternehmen gegen Flutschäden versichert sind, muss ein Teil der Kosten zur Beseitigung der Schäden demnach nicht von den Unternehmen getragen werden, sondern wird von Versicherungen übernommen.

Die Abbildung von Schäden durch Extremwetterereignisse läuft einer herkömmlichen gesamtwirtschaftlichen Modellierung zum Teil entgegen: Während dort Kapitalstöcke dem Bedarf nach Produktionsmitteln und diese der zu erwartenden Nachfrage und den Marktsignalen folgen, müssen nun Teile eines Unternehmens außer Betrieb gestellt werden und können teilweise innerhalb desselben Jahres auch nicht ersetzt werden. Heimische Produktion wird dabei kurzfristig durch Importe ersetzt, wenn die Infrastruktur zu beschädigt ist, um schnell in den ursprünglichen Zustand gebracht $\mathrm{zu}$ werden. Um beispielsweise Schäden eines Flusshochwassers an Produktionsstätten zu modellieren, wurde erstens der Kapitalstock des Maschinenbausektors um die zuvor in der Literaturrecherche identifizierten Schäden reduziert. Weitere Schäden beziehungsweise Auswirkungen, die nach dem gleichen Muster, jedoch auf Basis anderer Modellvariablen abgebildet wurden, sind Schäden an Wohngebäuden und an der Transportinfrastruktur, Auswirkungen auf die industrielle und landwirtschaftliche Produktion (letztere im Hinblick auf Hochwasser und Hitze) und auf den Katastrophenschutz. Die Wirkungen von Hitzewellen wurden abgebildet, indem Auswirkungen auf die 


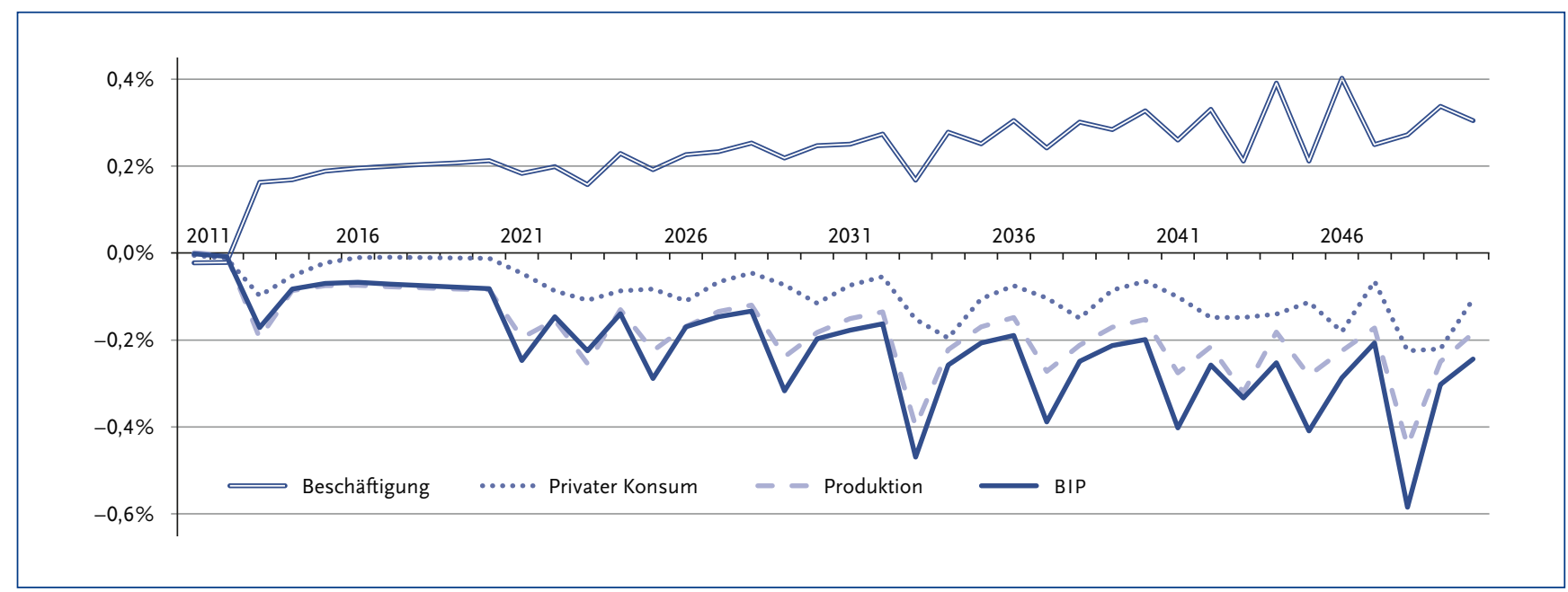

Abbildung 1: Wirtschaftliche Entwicklung, Differenz in Prozent zwischen Klima- und Referenzszenario, preisbereinigt

Energieproduktion, auf die Arbeitsproduktivität und auf den Schiffsverkehr Berücksichtigung fanden.

Mit dieser Vorgehensweise kann eine Simulation erstellt werden, die die Schäden durch Extremwetterereignisse und die gesamtwirtschaftlichen Anpassungsreaktionen bei Mengen, Löhnen und anderen Preisen berücksichtigt. Diese wird einer Welt gegenübergestellt, in der die Schäden nicht stattfinden, gewissermaßen eine kontrafaktische Welt ohne Klimawandel. Im Vergleich von Ergebnissen dieser Simulationen ergeben sich Aussagen zur Höhe des Einflusses von Extremwetterereignissen auf die Wirtschaftstätigkeit in Deutschland bis zum Jahr 2050. Wie oft treten Extremwetterereignisse auf? Das liegt außerhalb der Antworten, die mit einem gesamtwirtschaftlichen Modell gegeben werden können. Daher wurde hier die einfache Annahme so modelliert, dass Hochwasserereignisse alle zehn Jahre auftreten. Eine Ausnahme bildet die Dekade von 2040 bis 2050, in der zwei Flusshochwasser auftreten. Hitzewellen kehren alle vier Jahre wieder.

\section{Schaden für die deutsche Volkswirtschaft}

Insgesamt fallen die wirtschaftlichen Effekte von wiederkehrenden Hochwasserereignissen und Hitzewellen in Deutsch- land bis zum Jahr 2050 eher moderat aus. Der Gesamteffekt ist negativ auf einem niedrigen Niveau. In Abbildung 1 sind für vier ausgewählte Wirtschaftsindikatoren die Differenzen im Vergleich zwischen den beiden Simulationsläufen dargestellt. Die Schocks, die in regelmäßigen Abständen auf das Modell wirken, sind deutlich als Ausschläge zu erkennen. Der private Konsum, die Produktion und das Bruttoinlandsprodukt fallen durch die Extremwetterereignisse geringer aus als im kontrafaktischen Szenario, wobei sich dieser Effekt im Zeitablauf leicht verstärkt. Der private Konsum liegt infolge von Extremwetterereignissen bis zum Jahr 2020 unwesentlich und ab dem Jahr 2021 zwischen 0,1\% (1 Milliarde Euro) und 0,2\% (3 Milliarden Euro) niedriger. Die gesamtwirtschaftliche Produktion fällt infolge von Produktionsausfällen ebenfalls geringer aus: zwischen 0,1\% (3,5 Milliarden Euro) und 0,4\% (30 Milliarden Euro). Insgesamt liegt das Bruttoinlandsprodukt zwischen 0,1\% (1,75 Milliarden Euro) und 0,6\% (19,5 Milliarden Euro) niedriger. Hitzewellen machen die Beschäftigten unproduktiver, was das Modell als Zunahme von Beschäftigung abbildet. Um die gleiche Produktionsmenge zu erzeugen, werden mehr Arbeitskräfte benötigt als in der Referenz. Dadurch verringert sich der Lohn der Einzelnen, was sich wiederum im Rückgang des privaten Konsums widerspiegelt. Die stärksten

\begin{tabular}{|c|c|c|c|c|}
\hline Wirtschaftssektoren & $2011-2020$ & $2021-2030$ & $2031-2040$ & $2041-2050$ \\
\hline Land- und Forstwirtschaft, Fischerei & $0,1 \%$ & $0,0 \%$ & $0,1 \%$ & $0,1 \%$ \\
\hline Bergbau u. Verarbeitung v. Steinen u. Erden & $-0,2 \%$ & $-0,3 \%$ & $-0,3 \%$ & $-0,3 \%$ \\
\hline Verarbeitendes Gewerbe & $-0,1 \%$ & $-0,2 \%$ & $-0,2 \%$ & $-0,3 \%$ \\
\hline Energie- und Wasserversorgung & $0,0 \%$ & $-0,1 \%$ & $-0,1 \%$ & $-0,2 \%$ \\
\hline Bausektor & $0,1 \%$ & $0,2 \%$ & $0,3 \%$ & $0,3 \%$ \\
\hline Handel; Instandhaltung und Reparatur von Kfz und Gebrauchsgütern & $0,1 \%$ & $0,1 \%$ & $0,2 \%$ & $0,2 \%$ \\
\hline Gastgewerbe & $0,0 \%$ & $-0,1 \%$ & $-0,1 \%$ & $-0,2 \%$ \\
\hline Transport- und Kommunikationsdienstleistungen & $0,4 \%$ & $0,5 \%$ & $0,4 \%$ & $0,4 \%$ \\
\hline Kredit- und Versicherungswesen & $0,1 \%$ & $0,1 \%$ & $0,1 \%$ & $0,1 \%$ \\
\hline Übrige Dienstleistungen & $0,0 \%$ & $-0,1 \%$ & $-0,1 \%$ & $-0,1 \%$ \\
\hline
\end{tabular}

Tabelle 1: Bruttowertschöpfung, durchschnittliche Abweichungen per Dekade zwischen Klima- und Referenzszenario, in Prozent 
negativen Abweichungen sind in den Jahren 2033 und $2048 \mathrm{zu}$ beobachten - zwei Jahre, in denen ein Hochwasserereignis und eine Hitzewelle auftreten.

Um einen tiefer gehenden Einblick zu bekommen, ist es notwendig, nicht nur die aggregierten Effekte, sondern ebenfalls die Auswirkungen auf die unterschiedlichen Wirtschaftssektoren zu analysieren. Für einen besseren Überblick wurden die 59 Wirtschaftssektoren der Volkswirtschaftlichen Gesamtrechnung zu zehn Wirtschaftsbereichen zusammengefasst (siehe Tabelle 1).

Zusätzlich zu den annahmegestützten Einstellungen im Modell sind hier auch die jeweiligen Anpassungsreaktionen zu beobachten. Geht die Produktion in einem Wirtschaftsbereich zurück, fragt er auch weniger Vorleistungen nach, sodass der Initialeffekt verstärkt wird. Die Entwicklung der Bruttowertschöpfung auf sektoraler Basis im Klimaszenario vermittelt einen ersten Eindruck darüber, welche Sektoren am stärksten von Extremwetterereignissen und Anpassungsreaktionen betroffen sind. Auf Ebene der Wirtschaftsbereiche sind die Abweichungen natürlich im Einzelnen klein. Sie liegen zwischen $(-) 0,1 \%$ und (-)0,3\% mit Ausnahme des Transportsektors. Am stärksten negativ betroffen ist das verarbeitende Gewerbe, dessen Bruttowertschöpfung in der ersten Dekade 0,1\% (3,6 Milliarden Euro) und in der letzten Dekade 0,3\% (22 Milliarden Euro) geringer ausfällt als im Referenzlauf. Am stärksten positiv betroffen ist der Sektor der Transport- und Kommunikationsdienstleistungen, dessen Bruttowertschöpfung im Klimaszenario zwischen 0,4\% (6 Milliarden Euro) und 0,5\% (10 Milliarden Euro) höher verläuft als in der Referenz. Es folgen der Bausektor mit einer positiven Differenz von 0,1\% (1,7 Milliarden Euro) bis 0,3\% (4,5 Milliarden Euro) sowie der Handel, dessen Bruttowertschöpfung im Klimaszenario zwischen 0,1\% (2 Milliarden Euro) und 0,2\% (8 Milliarden Euro) höher liegt. Der Anstieg in den beiden letztgenannten Sektoren im Vergleich zum Referenzszenario löst den positiven Effekt im Transportsektor aus, da aufgrund der Schäden an Gebäuden, Anlagen, Infrastruktur und Deichen die Nachfrage nach Baumaterialien, nach der Entsorgung von Schutt sowie nach neuen Anlagen und Maschinen ansteigt. Analog entwickeln sich die Nachfrage nach Transportdienstleistungen und die Bruttowertschöpfung dieser Branche.

\section{Schlussfolgerungen für die Modellierung}

Extremwetterereignisse haben negative Folgen und zwingen einzelne Sektoren zu Überlegungen bezüglich ihrer Widerstandsfähigkeit gegenüber solchen Ereignissen. Gerade die leistungstragenden Branchen, wie das verarbeitende Gewerbe oder die Energiewirtschaft, können dabei in nicht unerheblichem Maße betroffen sein. Darüber hinaus gilt es, bei der Interpretation der Ergebnisse zu berücksichtigen, dass bislang nur Ereignisse innerhalb der Landesgrenzen berücksichtigt worden sind und das in einer Region in Europa, die eher gemäßigten klimatischen Veränderungen ausgesetzt sein wird.
Die Ergebnisse geben keinen Anlass, sich entspannt zurückzulehnen. Denn in extremer betroffenen Gebieten weltweit werden wichtige Vorleistungen und Inputs hergestellt, die die Wertschöpfungskette deutscher Unternehmen ganz empfindlich stören können. Darüber hinaus sind Wirtschaftszweige betroffen, die derzeit schon mit dem Umbau ihres Geschäftsmodells zu tun haben, allen voran die Energiewirtschaft.

Aus Forschungsgesichtspunkten hat die Untersuchung viele Lücken in den verfügbaren Daten offenbart, die dringend geschlossen werden müssen. Für eine sinnvolle Ausgestaltung auch von Anpassungsmaßnahmen müssen weitere Forschungsanstrengungen unternommen werden, um einen umfassenderen Einblick in die Auswirkungen von Extremwetterereignissen auf Deutschland zu bekommen. Detaillierte Kenntnisse über die Vulnerabilität eines Wirtschaftszweigs, auch stärker detailliert nach Regionen, sind im Hinblick auf wirtschaftspolitische Implikationen entscheidend. Darüber hinaus könnte der Zugang zu umfassenderen und detaillierteren Daten bezüglich der Schäden und Kosten, die aus Extremwetterereignissen in Deutschland resultieren, die Simulationsergebnisse verbessern.

\section{Literatur}

Ahlert, G./Distelkamp, M./Lutz, C./Meyer, B./Mönnig, A./Wolter, M.I. (2009): Das IAB/INFORGE-Modell. In: Schnur, P./Zika, G. (Hrsg.): Das IAB/ INFORGE-Modell. IAB-Bibliothek 318, S. 15-175.

IPCC (2014): Climate Change 2014: Impacts, Adaptation, and Vulnerability. Part B: Regional Aspects. Cambridge.

Lehr, U./Lutz, C./Edler, D. (2012): Green jobs? Economic impacts of renewable energy in Germany. In: Energy Policy 47, S. 358-364.

Lehr, U./Ulrich, P. (2014): Erneuerbar beschäftigt in den Bundesländern: Bericht zur aktualisierten Abschätzung der Bruttobeschäftigung 2013 in den Bundesländern. Osnabrück.

Lutz, C./Lehr, U. (2014): Macroeconomic Effects of Renewable Energy and Energy Efficiency Policies with a Focus on Germany. In: Bernhard, L./ Semmler, W. (eds.): The Oxford Handbook of the Macroeconomics of Global Warming. Oxford.

Münchner Rück (2013): Naturkatastrophen in Deutschland 1970-2013. Januar 2013.

Prognos, GWS, EWI (2010): Energieszenarien für das Energiekonzept. Basel. Rahmstorf, S./Schellnhuber, H.J. (2006): Der Klimawandel. München.
AUTOREN + KONTAKT

Dr. Ulrike Lehr arbeitet bei der Gesellschaft für Wirtschaftliche Strukturforschung im Bereich Energie und Klima.

Gesellschaft für Wirtschaftliche Strukturforschung, GWS mbH, Heinrichstr. 30, 49080 Osnabrück. E-Mail: lehr@gws-os.de

Anne Nieters arbeitet bei der Gesellschaft für Wirtschaftliche Strukturforschung im Bereich Energie und Klima.

Gesellschaft für Wirtschaftliche Strukturforschung, GWS mbH, Heinrichstr. 30, 49080 Osnabrück. E-Mail: nieters@gws-os.com
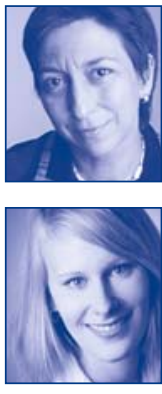